FORMATION Formation emploi

Revue française de sciences sociales

120 | Octobre-Décembre 2012

Parcours de formation : la recomposition des ségrégations

\title{
L'impact des ségrégations territoriales et universitaires sur les parcours étudiants : la filière AES
}

The impact of territorial and academic segregation on student trajectory: a study of a French « Social and Economic Administration » course

Einfluss der gebiets- und universitätsabhängigen Segregation auf die studentische Laufbahn im Studiengang AES

El impacto de las segregaciones territoriales y universitarias en los itinerarios estudiantiles: la orientación AES

Sandrine Nicourd, Olivia Samuel et Sylvie Vilter

\section{(2) OpenEdition Journals}

Édition électronique

URL : http://journals.openedition.org/formationemploi/3792

DOI : 10.4000/formationemploi.3792

ISSN : 2107-0946

Éditeur

La Documentation française

Édition imprimée

Date de publication : 31 octobre 2012

Pagination : $57-74$

ISSN : 0759-6340

Référence électronique

Sandrine Nicourd, Olivia Samuel et Sylvie Vilter, «L'impact des ségrégations territoriales et universitaires sur les parcours étudiants : la filière AES », Formation emploi [En ligne], 120 | OctobreDécembre 2012, mis en ligne le 15 février 2013, consulté le 30 octobre 2020. URL : http:// journals.openedition.org/formationemploi/3792; DOI : https://doi.org/10.4000/formationemploi.3792 


\section{L'impact des ségrégations territoriales et universitaires sur les parcours étudiants : la filière AES}

SANDRINE NiCOURD

Sociologue, maitre de conférences en sociologie à l'université de Versailles-St-Quentin et membre du laboratoire Printemps (Professions, Institutions, Temporalités - UMR UVSQ CNRS)

Olivia Samuel

Démographe, maître de conférences à l'université de Versailles St Quentin, membre du laboratoire Printemps (Profession, Institutions, Temporalités - UMR UVSQ CNRS) et chercheuse associée à l'INED (Institut national d'études démographiques).

SYLVIE VILTER

Maître de conférences en économie à l'université de Versailles St Quentin et membre du laboratoire Printemps (Professions, Institutions, Temporalités - UMR UVSQ CNRS)

Résumé

L'impact des ségrégations territoriales et universitaires sur les parcours étudiants : la filière AES

Les processus de ségrégation sociale sont rarement abordés à l'échelle des universités. À partir d'une recherche composée d'une enquête qualitative et d'une enquête quantitative réalisées sur deux universités de la région parisienne, cet article propose une analyse de deux formes de ségrégations, territoriale et universitaire. Les conditions de socialisation au sein des deux établissements pour la même filière AES (Administration économique et sociale) mettent en évidence les modalités de recomposition de la population étudiante entre la première et la dernière année de licence.

Mots clés : Inégalité sociale, université, approche locale, socialisation, AES - Administration Economique et Sociale - filière de formation, Ile-de-France

Abstract

The impact of territorial and academic segregation on student trajectory: a study of a French « Social and Economic Administration » course

Processes of social segregation are rarely addressed on the scale of whole universities. This paper is based on two surveys, a qualitative one and a quantitative one, of two universities of the Paris region. Its aim is to offer an analysis of two types of segregation: 
territorial segregation and academic segregation. Socialization processes within these two establishments, for the same "Social and Economic Administration" course, show how the student body is reconstructed between the first and third year of university.

Keywords : Social inequality, university, local approach, socialisation, economic and social administration, training stream, Ile-de-France

Journal of Economic Literature: I 21, I 23

Traduction : Auteurs

L'université française est marquée, depuis trois décennies, par de profonds changements. Aux réformes structurelles (réforme Bayrou, $\mathrm{LMD}^{1}$ ), se greffent des transformations liées au public étudiant et à l'arrivée massive de "nouveaux étudiants » issus des milieux populaires et souvent titulaires de bac technologiques ou professionnels (Albouy, Tavan, 2007 ; Convert, 2010). Les formations non sélectives, et en particulier celles des disciplines littéraires ou de sciences humaines et sociales, ont été particulièrement concernées par cette recomposition étudiante. Inversement, la clôture sociale des formations sélectives semble être toujours aussi puissante, la démocratisation scolaire a peu touché les grandes Ecoles en comparaison de l'université (Euriat et Thélot, 1995). Les universités ont été cependant diversement affectées par la démocratisation scolaire. Les antennes universitaires de province, ou certaines universités implantées dans des territoires paupérisés, ont été confrontées plus que les autres à la prise en charge du nombre croissant de ces nouveaux bacheliers (Beaud, 2002 \& 2008 ; Felouzis, 2001).

Plusieurs auteurs ont rendu compte des effets de la démocratisation scolaire qui, loin d'avoir seulement permis une augmentation quantitative du nombre d'élèves dans les études secondaires (démocratisation quantitative), a également eu pour conséquence de creuser des écarts entre les types de bac (généraux, technologiques, professionnels) et de spécialiser un certain nombre de cursus (Prost, 1986). Les élèves des catégories populaires ont pu accéder à certaines voies d'études secondaires longues, mais pas n'importe lesquelles. La démocratisation qualitative, qui aurait dû réduire les inégalités de parcours entre élèves de milieux sociaux différents, n’a pas eu lieu.

Dans l'enseignement supérieur, le constat n'est pas très éloigné. On observe plutôt un renforcement de la spécialisation sociale des filières (Merle 2002 ; Sautory, 2007). La composition socio-scolaire des filières du supérieur mérite un examen précis : il s'agit, notamment, de rendre compte des modalités de différenciation sociale des filières pour en saisir les conséquences sur les trajectoires étudiantes.

1. La « réforme Bayrou " sur l’université, en 1997, proposait une organisation du premier cycle en semestres. La réforme LMD (2006) réorganise l'offre universitaire selon une harmonisation européenne en trois niveaux : licence, master, doctorat. 
Peut-on parler de filières ségréguées ? Plus largement, l'article propose une réflexion sur deux formes de ségrégation : d'une part, la spécialisation sociale des filières et d'autre part, la différenciation sociale des établissements d'enseignement supérieur au sein d'un territoire. La ségrégation peut être entendue comme un processus de structuration sociale de l'espace et des groupes sociaux qui se traduit par les chances inégales d'accès aux biens matériels et symboliques offerts par un lieu ou un espace urbain (ou inégale distribution des groupes sociaux dans l'espace). Dans cette perspective, la ségrégation se définit comme " toute forme de regroupement spatial associant des populations défavorisées à des territoires défavorisés" (Grafmeyer, 1966). La disparité renvoie de fait à un processus de hiérarchisation, de classement.

La ségrégation s'oppose ainsi à la différenciation, l'hétérogénéité sociale. Eric Maurin (2004) précise qu' "il existe un verrouillage général, durable et silencieux des destins sociaux" par les processus de ségrégation. L'enjeu est donc d'identifier les conséquences de contextes sociaux plus ou moins ségrégués sur les parcours étudiants.

Dans cet article, nous nous interrogeons sur l'effet conjugué des inégalités territoriales et des inégalités entre filières de formation sur les trajectoires étudiantes. Autrement dit, nous tentons de comprendre comment se combinent les effets de la ségrégation territoriale et de la ségrégation des filières universitaires sur les parcours étudiants en comparant deux universités situées dans des départements socio-économiquement contrastés de la région parisienne. La ségrégation est mesurée par la surreprésentation, dans les populations concernées (territoire, filière), de caractéristiques socio-économiques, sociales et scolaires particulières. Ainsi, nous pourrons contribuer au débat sur les processus concrets de démocratisation au sein des universités françaises en combinant un regard quantitatif longitudinal et une approche qualitative dans l'analyse des processus de socialisation des étudiants. La socialisation est ici entendue comme un processus d'ajustement entre les caractéristiques d'une trajectoire socio-biographique et les dimensions d'un système social avec des contraintes institutionnelles et cognitives spécifiques (Dubar, 2010).

Dans un premier temps, nous examinerons les principales caractéristiques territoriales et de formation des populations étudiées afin d'objectiver les processus ségrégatifs à l'œuvre ; dans un deuxième temps, nous étudierons les effets de ces phénomènes sur les parcours d'études dans chacune des deux universités, tout en rendant compte des conditions de socialisation des étudiants pour comprendre ces trajectoires de formation contrastées.

L'enquête s'appuie sur la comparaison de deux universités, l'une située dans un département favorisé, les Yvelines, et l'autre dans un département défavorisé, la Seine-St-Denis. Chacun de ces deux établissements propose de nombreuses formations ; cependant, nous avons choisi de nous centrer sur la filière AES (Administration économique et sociale), car elle est probablement la plus emblématique de la démocratisation scolaire. Elle accueille massivement les bacheliers issus des milieux populaires et les titulaires des bac technologiques et professionnels (Montfort, 2003 ; Nicourd et alii, 2009). 


\section{Encadré 1}

L'enquête

La recherche " Trajectoire d'études et insertion sociale des étudiants d'AES » (Administration économique et sociale) vise à comprendre les modes d'investissement des étudiants dans leurs études universitaires à partir d'un éclairage des différentes socialisations qui ont construit et construisent leur trajectoire. Elle s'intéresse en particulier à trois étapes clé : l'entrée à l'université, le parcours de formation et l'insertion professionnelle.

Dans le cadre de cet article, nous examinons principalement la question du parcours en licence.

Le protocole d'enquête mis en œuvre dans les universités Fac Ouest et Fac Nord (respectivement dans le département des Yvelines et dans le département de la Seine-St-Denis) s'articule autour du suivi d'une cohorte d'étudiants inscrits en $1^{\text {ère }}$ année dans la filière AES à la rentrée 2005-2006.

Pour mener à bien ce suivi longitudinal, plusieurs sources d'information sont utilisées : les données administratives des universités ainsi qu'une enquête par questionnaire et par entretiens semi-directifs auprès des étudiants. Lors de la première vague de l'enquête (année 1), les étudiants inscrits en 1 ère année de droit et d'économie ont également été interrogés à des fins comparatives.

Les données administratives permettent de reconstituer partiellement I'histoire de cette cohorte sur cinq années successives. La population initiale est composée de 676 étudiants inscrits en AES, 258 à Fac Ouest et 418 à Fac Nord.

L'enquête par questionnaire a été réalisée auprès de cette même cohorte d'étudiants. Le nombre de répondants au démarrage de l'étude était de 125 à Fac Ouest (48\% des inscrits en AES) et de 189 à Fac Nord ( $45 \%$ des inscrits). L'attrition de la cohorte au fil du temps est sensible.

En parallèle, une série d'entretiens annuels semi-directifs en face-à-face a été menée auprès d'un échantillon d'étudiants de la cohorte. Au total, 60 entretiens ont été réalisés sur les cinq années de suivi. Le suivi longitudinal qualitatif comporte toujours une attrition, notamment pour une population étudiante confrontée à de nombreux changements d'adresses. Au total, $20 \%$ des entretiens ont été réalisés au cours des cinq années, $40 \%$ ont été conduits pendant une période de deux ou trois années et $40 \%$ prennent la forme d'un récit rétrospectif sur les années d'études. Pour l'analyse des entretiens, nous avons procédé à une analyse thématique transversale qui nous a permis d'identifier des configurations récurrentes.

Cette phase qualitative a été complétée, dans les deux universités, par des entretiens réalisés auprès de quatorze enseignants et de trois personnels administratifs de la filière $A E S\left({ }^{*}\right)$ afin de comprendre la place prise par ces acteurs dans le processus de socialisation des étudiants.

$\left.{ }^{*}\right)$ : Cette partie de l'enquête a été réalisée par Isabelle Lacroix, laboratoire PRINTEMPS. Nous la remercions pour la lecture de cet article et ses suggestions. 
Encadré 2

Deux territoires, deux universités, une filière

L'enquête porte sur deux universités de la région parisienne. Fac Nord, située en Seine-St-Denis, est une université pluridisciplinaire de plus de 20000 étudiants, créée au début des années 70. Fac Ouest, dans les Yvelines, est de création plus récente (années 80 ) ; elle est également pluridisciplinaire et regroupe environ 15000 étudiants.

La filière AES (Administration économique et sociale), créée en 1973, a comme originalité de proposer une formation alliant plusieurs disciplines : le droit, les sciences économiques et les sciences sociales (principalement la sociologie, la psychologie et l'histoire).

Les deux universités offrent le même diplôme, la licence d'AES, avec des contenus voisins.

\section{Distance sociale et scolaire d'une université à l'autre}

La Seine-St-Denis est un département ségrégué au sein des départements d'Ile-de-France : les principaux indicateurs montrent en effet de forts contrastes et y pointent une concentration des difficultés socio-économiques. Et si l'on pousse la comparaison avec le département des Yvelines, les inégalités territoriales sont alors encore plus flagrantes.

Le taux de chômage des Yvelines est parmi les dix plus bas en France, et celui de la SeineSt-Denis l'un des plus élevés. La part des cadres est trois fois plus importante dans les Yvelines qu'en Seine-St-Denis et inversement, les ouvriers y sont deux fois moins nombreux. Les indicateurs socio-économiques de La Seine-St-Denis sont parmi les plus défavorables de tous les départements de France métropolitaine, contrairement aux Yvelines qui se situent à l'opposé du classement.

L'immigration en Ile-de-France est importante, et les Yvelines comme la Seine-St-Denis font partie des départements à forte proportion de population étrangère ; cependant, en Seine-St-Denis, celle-ci est 2,5 fois plus importante et moins souvent originaire des pays de l'Union européenne que dans les Yvelines.

Du point de vue du contexte de formation, les taux de scolarisation sont relativement élevés dans les deux départements. L'abondance de l'offre de formation en région parisienne y participe certainement ; toutefois, la Seine-St-Denis est en léger retrait par rapport aux Yvelines et accuse également un taux de réussite au bac nettement inférieur, quel que soit le type de bac considéré.

Ces données territoriales posées, rien d'étonnant à ce qu'elles conditionnent en grande partie la composition socio-économique du public étudiant dans chacun des départements. En effet, lorsqu'ils choisissent une université, les lycéens optent le plus souvent pour un établissement proche de leur domicile, qui est par ailleurs généralement celui de 
leurs parents. Ce choix est d'autant plus facilité que la $1^{\text {ère }}$ année universitaire est sectorisée et que pour les filières non sélectives, les nécessités de mobilité géographique sont réduites, l'offre universitaire étant assez homogène sur l'ensemble du territoire national.

Pour autant, la population étudiante des premiers cycles universitaires n'est pas le fidèle reflet de l'espace social et territorial auquel elle appartient. Tous les lycéens ne vont pas à l'université. D'une part, la sélection en amont du système scolaire élimine la frange la plus en difficulté sociale et scolaire; d'autre part, l'évitement universitaire par le choix d'une formation sélective détourne la frange la plus dotée. Les bacheliers généraux (et dans une moindre mesure les bacheliers technologiques) optent de plus en plus souvent pour des formations sélectives ou des écoles privées, contrairement aux bacheliers professionnels qui s'inscrivent plus fréquemment à l'université (Lemaire, 2010).

Au niveau national, Fac Ouest (les Yvelines) se situe dans la frange des universités les plus favorisées du point de vue de l'appartenance sociale de ses étudiants, alors que Fac Nord (Seine-St-Denis) est très nettement l'une des plus défavorisées². À l'image de son territoire, celle-ci accueille en lère année de Licence une population majoritairement d'origine populaire. Les résultats présentés ici, tirés de l'enquête par questionnaire auprès des étudiants d'AES, de droit et de sciences économiques, indiquent que la moitié des inscrits de première année à Fac Nord a au moins un de ses parents ouvrier ou employé (Tableau 1). Ce n'est le cas que d'un étudiant sur cinq à Fac Ouest où, inversement, un étudiant sur deux a au moins l'un de ses deux parents cadre.

Le niveau de diplôme des parents est tout autant polarisé : à Fac Ouest, un étudiant sur trois a des parents non bacheliers contre plus de la moitié des étudiants de Fac Nord. Cette université accueille donc massivement des jeunes en situation de promotion scolaire qui sont les premiers de leur lignée à accéder aux études supérieures.

Enfin, un troisième indicateur confirme la spécificité de Fac Nord : il concerne l'origine géographique des étudiants. Si la plupart sont nés en France, seul un quart des étudiants a ses deux parents nés en France contre les trois quarts à Fac Ouest. Les jeunes issus de l'immigration représentent la grande majorité des étudiants de Seine-St-Denis, une immigration principalement Maghrébine ou d'Afrique sub-saharienne. Ces caractéristiques sociodémographiques sont loin d'être neutres sur les parcours scolaires et universitaires. Les résultats issus du panel sur les élèves entrés en $6^{\text {ème }}$ en 1995 montrent l'effet sensible de l'origine sociale, culturelle et géographique sur l'obtention du bac et des diplômes universitaires, outre les facteurs proprement scolaires (Lemaire, 2012).

2. Au niveau national, en licence, la part des enfants de cadre est de l'ordre d'un tiers et la part des enfants d'ouvrier ou d'employé est de un quart. Pour les données nationales, se référer aux données de l'Observatoire des inégalités et à l'Enquête conditions de vie, 2010, de l'OVE. 
Tableau 1

Caractéristiques sociodémographiques et scolaires des étudiants de 1 ère année de droit, sciences économiques et AES (Administration économique et sociale) en 2005-06, selon l'établissement d'inscription (\%)

\begin{tabular}{|c|c|c|c|}
\hline Indicateur & Modalités & $\begin{array}{l}\text { Fac Ouest } \\
\text { (Yvelines) }\end{array}$ & $\begin{array}{c}\text { Fac Nord } \\
\text { (Seine-St-Denis) }\end{array}$ \\
\hline \multirow{2}{*}{$\begin{array}{l}\text { PCS des parents } \\
\text { Au moins un des } 2 \text { parents.... }\end{array}$} & ... cadre & 49 & 18 \\
\hline & $\ldots$ ouvrier ou employé $(1)$ & 19 & 50 \\
\hline \multirow[t]{2}{*}{ Niveau d'études des parents } & $\begin{array}{l}\text { Au moins un des } 2 \text { parents de niveau } \\
\mathrm{Bac}+1 \text { ou plus }\end{array}$ & 57 & 26 \\
\hline & Les 2 parents sans le niveau bac & 33 & 54 \\
\hline Pays de naissance de l'étudiant & Né en France & 91 & 75 \\
\hline Pays de naissance des parents & Les deux parents nés en France & 72 & 26 \\
\hline \multirow{3}{*}{ Type de bac } & Bac général & 79 & 52 \\
\hline & Bac technologique & 18 & 36 \\
\hline & Bac professionnel & 3 & 9 \\
\hline Age au bac & A l'heure ou en avance & 65 & 50 \\
\hline Mention au bac & Avec mention & 30 & 16 \\
\hline
\end{tabular}

Source : Enquête Cohorte AES, 2005-06.

(1) Si un parent est cadre et l'autre est ouvrier/employé, alors l'étudiant est classé dans la catégorie « au moins un des deux parents cadre $"$.

PCS : profession et catégorie sociale.

Les inégalités qui ressortent de ce rapide portrait sociodémographique des étudiants des deux universités ne sont que renforcées lorsque l'on examine le bagage scolaire acquis au seuil des études supérieures. Les étudiants de première année de droit, de sciences économiques et d'AES de Fac Nord sont moins souvent titulaires d'un bac général, d'une mention au bac, ils ont en revanche accumulé du retard scolaire du fait de redoublements. En moyenne, leur performance scolaire est moindre que celle des étudiants de Fac Ouest. C'est une donnée importante car plus que le capital économique, ce facteur est le plus déterminant dans le processus de réussite dans les études supérieures (Duru-Bellat et Kieffer, 2008). 


\section{D'une inégalité entre territoires à une inégalité entre filières : une relation complexe}

Il s'agit maintenant d'examiner l'existence d'une éventuelle forme ségrégative " redoublée ", entre territoires et entre filières de formation. En d'autres termes, les inégalités socio-économiques entre les départements des Yvelines et de la Seine-St-Denis, qui se répercutent sur leurs publics étudiants, sont-elles renforcées par des inégalités socio-économiques et scolaires entre les filières de formation au sein des universités?

La filière AES se distingue-t-elle des autres formations, en particulier de droit et d'économie, qui sont par ailleurs les principales disciplines du cursus d'AES ? Cette filière est en effet particulière. À visée professionnelle (concours de la fonction publique), l'AES s'est révélée très attractive pour les bacheliers qui espéraient une promotion sociale via des études supérieures, avec un horizon déterminé, de moyen terme, dans le secteur protégé du marché du travail (le fonctionnariat) (Dumoulin, Filhon, 2008 ; Filhon, 2010). Peu à peu, cette formation a pris les contours d'une filière dépréciée, s'inscrivant dans le mouvement de la "démocratisation ségrégative " de l'institution scolaire (Merle, 2000). Cette ségrégation se traduit à la fois d'un point de vue objectif, sur la base des caractéristiques socio-scolaires des étudiants d'AES et aussi, comme nous le verrons par la suite, selon la considération accordée et le regard porté par les enseignants sur cette filière. Pourtant, ce processus ségrégatif ne s'opère pas de la même façon selon les établissements et leurs contextes territoriaux.

Pour examiner si les différences entre établissements observées plus haut se doublent de différences intra-établissement entre les filières de formation, nous avons comparé les profils étudiants en $1^{\text {ère }}$ année de licence d'AES à ceux de $1^{\text {ère }}$ année de droit et de sciences économiques, respectivement à Fac Nord et à Fac Ouest.

Le tableau 2 présente un indice qui rend compte des écarts entre l'AES et les deux autres filières. Plus cet indice s'éloigne de 1, valeur représentative de l'absence d'écart, et s'approche de 0 , moins l'AES est dans une situation favorable au regard des critères sélectionnés (avoir un bac techno ou pro, ne pas être mentionné au bac, avoir au moins une année de retard à l'obtention du bac, avoir ses deux parents non bacheliers, avoir ses deux parents ouvriers ou employés). De façon presque systématique, les différences socio-scolaires entre filières sont assez peu prononcées, voire absentes pour ce qui est de la mention au bac et de l'origine sociale des étudiants. En revanche, à Fac Ouest, les étudiants de $1^{\text {èr }}$ année d'AES sont plus souvent titulaires d'un bac pro ou techno, accusent plus souvent un retard scolaire et sont d'origine sociale plus modeste que les étudiants de droit et d'économie. Ces résultats permettent de conclure à une différence intra-établissement importante à Fac Ouest et assez faible à Fac Nord. 
Tableau 2

Indice d'écart à l'AES (Administration économique et sociale). Etudiants de 1 ère année de droit, sciences économiques et AES en 2005-06, selon l'établissement d'inscription

\begin{tabular}{|l|l|c|c|c|c|}
\cline { 3 - 7 } \multicolumn{2}{c|}{} & \multicolumn{2}{c|}{ Fac Ouest (Yvelines) } & \multicolumn{2}{c|}{ Fac Nord (Seine-St-Denis) } \\
\hline Indicateur & \multicolumn{1}{c|}{ Modalité } & Eco/AES & Droit/AES & Eco/AES & Droit/AES \\
\hline Type de bac & Bac techno + bac Pro & 0,24 & 0,39 & 0,85 & 0,88 \\
\hline Mention au bac & Sans mention & 0,88 & 0,96 & 1,00 & 0,93 \\
\hline Age au bac & Retard d'au moins 1 an & 0,57 & 0,85 & 0,76 & 0,80 \\
\hline $\begin{array}{l}\text { Niveau d'études des } \\
\text { parents }\end{array}$ & $\begin{array}{l}\text { Les deux parents ont un } \\
\text { niveau inférieur au bac }\end{array}$ & 0,60 & 0,43 & 0,70 & 0,82 \\
\hline PCS (*) des parents & $\begin{array}{l}\text { Les deux parents } \\
\text { ouvriers/employés (et/ou } \\
\text { inactifs) }\end{array}$ & 0,73 & 0,42 & 1,00 & 0,94 \\
\hline
\end{tabular}

Source : Enquête Cohorte AES, 2005-06.

Indice : rapport de pourcentage. Si le rapport est égal à 1, l'écart entre les deux filières est nul, si le rapport est inférieur à 1, l'AES a un pourcentage supérieur à celui du droit ou de l'économie.

Lecture : un indice de 0,24 (8,8\% de bacs techno et pro en L1 économie à Fac Ouest / 40,8\% de bacs techno et pro en L1 AES à Fac Ouest) indique que la filière économie a moins d'étudiants titulaires d'un bac techno ou pro que la filière AES.

$\left({ }^{*}\right)$ PCS : profession et catégorie sociale.

On est donc en présence d'un processus de ségrégation territoriale, faiblement renforcée par une ségrégation de filières en première année à Fac Nord. De faibles écarts socioscolaires sont observés en défaveur de l'AES (un peu plus de bacheliers non généraux, de non-mentionnés, de retardataires au bac et d'étudiants d'origine culturelle modeste) qui pourraient indiquer une forme de distinction de l'élite scolaire populaire vers les filières plus prestigieuses du droit et de l'économie. Toutefois, ce phénomène est bien plus massif à Fac Ouest, où prévaut une spécialisation socio-scolaire des filières de formation. Par rapport aux étudiants de droit et de sciences économiques, ceux d'AES sont bien plus souvent titulaires d'un bac technologique ou professionnel, ont davantage redoublé pendant leur scolarité et sont plus souvent issus des milieux peu favorisés.

Dans cette université, on peut faire l'hypothèse que les étudiants culturellement et économiquement dotés et détenteurs d'un bon capital scolaire se détournent - ou sont détournés par leurs parents et leur entourage - de formations telles que l'AES, peu prestigieuses, non élitistes et soupçonnées de faire courir un risque de déclassement. Autrement dit, le niveau socio-économique plus élevé de Fac Ouest va de pair avec une hiérarchisation socio-scolaire des formations, l'AES représentant le bas de cette hiérarchie. En effet, ce qui attire les jeunes des milieux populaires (notamment la sécurité des emplois publics de niveau intermédiaire, voir à ce sujet Filhon, 2010 ; Hugrée 2009) fait plutôt fuir ceux des milieux dotés, qui aspirent à travailler soit dans le privé, soit dans les emplois publics de la 
haute administration. Par ailleurs, l'ouverture socio-scolaire dans cette filière, qui accueille rappelons-le de nombreux élèves des séries technologiques, peut avoir un effet repoussoir pour les étudiants des classes moyennes et supérieures.

La spécialisation socio-scolaire de la filière AES, forte à Fac Ouest, plus modérée à Fac Nord, fait l'objet d'un discours particulièrement stigmatisant de la part des enseignants qui y font cours. Loin d'être homogène, le groupe socio-professionnel des enseignants du supérieur est différencié dans ses statuts, dans ses propres trajectoires de formation et également dans les conceptions de la relation pédagogique. Ainsi, l'enquête menée auprès des enseignants révèle que la majorité d'entre eux n'hésite pas à parler d'une filière dépréciée, déplorant régulièrement le niveau inférieur de ces étudiants en comparaison des autres et leurs décalages culturels dans leur façon de parler, voire de se comporter. En outre, la formation d'AES ne bénéficie pas de la spécialisation disciplinaire qui permettrait aux enseignantschercheurs d'y cultiver et d'y développer leurs recherches. Composée autour d'au moins trois disciplines différentes (droit, économie et sociologie), cette filière ne permet d'ailleurs pas non plus aux étudiants de bénéficier d'une socialisation par les matrices disciplinaires qui agissent comme autant de filtres socio-scolaires et sociocognitifs (Bodin, Millet, 2011). Les attentes des enseignants sont également hétérogènes, tant en matière de méthodes de travail que de répertoires cognitifs. Dans cette filière, la surreprésentation d'enseignants vacataires et la composition socio-culturelle de la population étudiante conduisent parfois des enseignants statutaires à refuser d'y réaliser une partie de leur service. Ces faits, révélateurs d'un processus de distinction fondé sur une hiérarchie de la valeur académique des formations au sein de l'université, favorisent une dépréciation symbolique de l'AES :

"Ils sont très enfantins, c'est un désert culturel ici. Les deux premières années sont là pour faire le tri entre ceux qui ont des capacités intellectuelles et les autres.» (Enseignant statutaire en AES)

"Je ne retrouve pas chez eux la curiosité que j'avais quand j'étais étudiant. C'est à se demander ce quils font à l'université. " (Enseignante AES)

Quelques enseignants, moins nombreux, vont à l'inverse investir leur rôle d'une responsabilité sociale : ils développent des approches pédagogiques plus interactives, des modalités relationnelles visant à moins assigner les étudiants à des positions d'infériorité culturelle. On retrouve notamment cette démarche chez d'anciens enseignants du secondaire, ayant travaillé en zone d'éducation prioritaire.

Si les étudiants d'AES ne perçoivent pas immédiatement la hiérarchisation entre les filières, notamment en première année, ils intériorisent ensuite qu'ils sont dans une filière peu reconnue au sein de l'université :

"Ici, en AES, je me suis rendu compte que pour beaucoup de profs, c'est un second travail donc c'est pas leur vraie vie, ils sont avocats ou bien enseignants ailleurs et puis du coup, on sent quils sont de passage, on les sent pas impliqués et puis sinon, y a les grands profs, ceux qui font les amphis, ceux là, ils arrivent et ils partent tout de suite à la fin du cours. En AES, on a un peu de culture en surface sur plusieurs sujets. " (Etudiant, L2 d'AES) 
"Les étudiants en AES ils nous tirent vers le bas, on voudrait aller plus loin et la prof est obligée de s'ajuster à leur niveau. " (Etudiant en L3 de sociologie, suivant un cours commun avec les AES)

Comment se déroulent les parcours des étudiants en AES dans un tel contexte ? Quelles sont les logiques qui expliquent l'évolution de la population étudiante et sa recomposition entre la première et la troisième année ?

\section{Une homogénéisation des niveaux scolaires, 2 un maintien des disparités socio-économiques}

Point de départ du cursus en AES, l'entrée à l'université en 1ère année inaugure un cheminement non rectiligne pour la majorité des étudiants. Les trajectoires sont marquées par des abandons, des réorientations, des redoublements ou des échecs pour près des quatre cinquièmes des étudiants. Bodin et Millet (2011) insistent à juste titre sur le fait que l'échec et l'abandon des études en lère année constituent davantage l'exception que la règle, mais que le taux d'évaporation, via notamment les changements de voies, est très élevé au cours de la lère année. C'est un trait commun à presque toutes les formations universitaires. Toutefois, l'AES est un peu plus concerné par les sorties sans diplôme de l'enseignement supérieur (38\% ${ }^{3}$, Lemaire, 2007) et par le taux d'abandon après une année d'étude (50\%, MEN-MESR, 2007).

Le suivi de la cohorte à partir des données administratives permet d'établir que seuls $16 \%$ des inscrits en L1 d'AES à Fac Nord sont trois ans plus tard en L3 d'AES contre $22 \%$ à Fac Ouest. Que se passe t-il dans cet intervalle de trois ans ? Comment se construisent les parcours? Quelles différences observe-t-on entre les deux universités ?

Le déroulé de la lère année présente d'assez faibles différences d'une université à l'autre. La part des défaillants - des étudiants ne se présentant pas aux examens - est équivalente (autour de $55 \%$ ) alors que la proportion de réussite en L1 est légèrement supérieure à Fac Ouest (41 \%) qu’à Fac Nord (37 \%). De façon similaire dans les deux établissements, seul un bachelier professionnel sur vingt valide sa lère année, contre un bachelier technologique sur cinq et près de trois bacheliers des séries générales sur cinq. Les étudiants les moins armés scolairement, les bacheliers professionnels et, dans une moindre mesure, les étudiants issus des séries technologiques, décrochent rapidement. Certains de ces étudiants qui étaient en attente d'une place dans une formation sélective connaissent des départs anticipés ; cependant, ce décrochage résulte aussi d'un processus de sélection scolaire, avec d'éventuelles réorientations dans d'autres filières ou vers des écoles privées, ou est le fruit d'une auto-exclusion liée au sentiment de ne pas être "à sa place».

3. Résultat issu du suivi des élèves entrés en Gème en 1989 puis inscrits à l'université après leur bac (panel ministère de l'Éducation nationale). 
"Quand je vais à la fac, je m’y retrouve pas. J'ai raté quelques cours en amphi parce que je travaillais à des horaires que je n'ai pas choisis et puis je ne comprends pas ce qui se passe, pourquoi le prof parle de ça ou de ça. Je ne vois pas le lien avec le lycée, à quoi sert tout ça, jai l'impression de perdre mon temps. " (étudiante, année 1, AES, Fac Nord)

Au fil des trois années après l'entrée en AES, la déperdition se poursuit avec des modalités toutefois différentes dans les deux universités. À Fac Ouest, selon un modèle assez courant, une part importante des étudiants ne s'est pas réinscrit dans la formation (ni à l'université) à l'issue de la première année (50\%). Nombreux sont les étudiants qui cumulent leurs études avec de "petits boulots", aussi la conscience de ces conditions socio-économiques est-elle très forte en cas de difficultés ou d'échec. Dans les moments de doutes sur leur engagement dans leurs études, les étudiants sont tiraillés entre leur statut de salarié (le plus souvent précaire) et leur statut d'étudiant. Dès lors, ils ont tendance à se réfugier vers une activité salariée qui leur parait plus utile à court terme, notamment parce qu'elle répond à leurs incertitudes financières.

Selon l'enquête Cohorte AES 2005-06, 46 \% des étudiants de Fac Ouest et $35 \%$ de ceux de Fac Nord ont déclaré avoir un emploi. La durée de travail hebdomadaire est supérieure à neuf heures pour la moitié des étudiants de Fac Ouest et les trois quarts de Fac Nord.

À Fac Nord, la plus forte proportion de réinscrits à l'issue de la $1^{\text {ère }}$ année (45\%) en première année s'accompagne d'un taux de redoublement deux fois plus élevé. Une partie des étudiants de Fac Nord ne prend donc pas le risque d'abandonner les études engagées et tente de s'accrocher, dans une logique de précaution, faute d'une alternative viable d'études ou d'emploi, mais aussi dans une logique de continuité d'une trajectoire scolaire qui a ouvert des perspectives inédites dans le milieu d'origine. En effet, bien des étudiants de Fac Nord sont les premiers de leur famille à avoir décroché le bac. Leurs études secondaires accomplies, parfois de façon chaotique, puis l'obtention du bac ont conduit ces «enfants de la démocratisation » à aspirer à une promotion sociale par l'enseignement supérieur (Beaud, 2002 ; 2008 ; Hugrée, 2009). Ils s'accrochent, conscients que peu d'autres voies leur sont ouvertes et qu'ils ont déjà expérimenté cette relative fermeture des horizons possibles : $38 \%$ d'entre eux ont demandé une autre formation à la sortie du lycée, en général en STS ou en IUT (respectivement sections de technicien supérieur et instituts universitaires de technologie), mais n’y ont pas été admis.

Pour les étudiants toujours inscrits en AES après une $1^{\text {ère }}$ année, la fréquence des départs de l'université est encore élevée à Fac Nord (31\% contre $13 \%$ à Fac Ouest). Certains de ces étudiants ont procédé à deux inscriptions en première année, et face à un nouvel échec, quittent l'université.

À mesure que s'opère le processus de sélection, d'auto-sélection et de réorientation des étudiants, la cohorte initiale se transforme. Les nombreux étudiants qui quittent l'université n'ont pas le même profil socio-scolaire que ceux qui restent, en particulier à Fac Nord. Peu à peu, les étudiants au parcours scolaire moins " performant " disparaissent : ceux issus des bacs technologiques ou professionnels et ceux qui ont accumulé un retard scolaire. 
Au bout de trois années, le profil scolaire des étudiants d'AES présents dans les deux universités a fortement changé, contrairement au profil socio-économique. Sur le plan du capital scolaire, l'écart entre les deux universités s'amenuise et les étudiants de 3ème année de licence AES ont un profil quasiment similaire d'une université à l'autre. Ainsi, en $1{ }^{\text {ère }}$ année de licence, $31 \%$ des étudiants de Fac Nord cumulaient un bac non généraliste, une mention passable au bac et un retard scolaire, contre $14 \%$ à Fac Ouest. En $3^{\mathrm{ème}}$ année, cette proportion est d'environ $10 \%$ dans les deux universités (enquête par questionnaire). Les étudiants les plus dépourvus des pré-requis attendus par l'université ont en grande partie disparu.

Mais cette homogénéisation des niveaux scolaires, trois ans après l'entrée à l'université, s'accompagne d'un maintien des différences socio-économiques. Fac Nord accueille toujours en AES un public étudiant majoritairement issu des classes populaires en $3^{\text {ème }}$ année de licence (environ la moitié des étudiants), alors que Fac Ouest concentre des étudiants issus des classes moyennes et supérieures.

Tableau 3

\section{Facteurs explicatifs de l'obtention de L1 et de L3 sans redoublement dans chaque établissement (odd ratios)}

\begin{tabular}{|c|c|c|c|c|}
\hline & \multicolumn{2}{|c|}{$\begin{array}{c}\text { Résultats 2005-06 } \\
\text { Obtention de L1 ( }{ }^{1} \text { ère année) }\end{array}$} & \multicolumn{2}{|c|}{$\begin{array}{c}\text { Résultats 2007-08 } \\
\text { Obtention de L3 (3 } 3^{\text {ème } a n n e ́ e) ~}\end{array}$} \\
\hline & $\begin{array}{l}\text { Fac Ouest } \\
\text { (Yvelines) }\end{array}$ & $\begin{array}{c}\text { Fac Nord } \\
\text { (Seine-St-Denis) }\end{array}$ & $\begin{array}{l}\text { Fac Ouest } \\
\text { (Yvelines) }\end{array}$ & $\begin{array}{c}\text { Fac Nord } \\
\text { (Seine-St-Denis) }\end{array}$ \\
\hline \multicolumn{5}{|l|}{ Sexe réf: femme } \\
\hline Homme & ns & 1,5 & ns & ns \\
\hline \multicolumn{5}{|c|}{ Age bac réf: à l'heure } \\
\hline En retard & $-2,0$ & ns & ns & ns \\
\hline \multicolumn{5}{|c|}{ Type de bac réf: bac général } \\
\hline Bac techno/pro & $-2,9$ & $-4,5$ & 0,3 & $-5,3$ \\
\hline \multicolumn{5}{|c|}{ PCS réf: cadres, prof. intermédiaires, indépendants } \\
\hline Employés et ouvriers & ns & 0,6 & ns & 0,5 \\
\hline Inactifs / Sans réponse & ns & ns & ns & \\
\hline \multicolumn{5}{|c|}{ Lieu de naissance réf: département de l'université et départements limitrophes } \\
\hline Autres départements & ns & ns & ns & ns \\
\hline Etranger & ns & ns & ns & ns \\
\hline
\end{tabular}

Source : Fichiers administratifs.

Effectifs : 227 étudiants en L1 à Fac Ouest - 418 étudiants en L1 à Fac Nord.

Lecture : La probabilité d'obtention de L1 est 2,9 fois moindre $(1 / 2,9=0,34)$ pour les étudiants de Fac Ouest ayant obtenu leur bac en techno ou pro par rapport aux titulaires d'un bac général.

L'individu de référence est une étudiante ayant obtenu un bac général, « à l'heure », dont les parents sont cadres, prof. inter. ou indépendants, née dans le département de son université ou les départements limitrophes.

Significativité : toutes les valeurs présentées dans le tableau sont significatives au seuil de $5 \%$.

Lieu de naissance : pour Fac Ouest : Yvelines et départements limitrophes, pour Fac Nord : Seine-St-Denis et départements limitrophes.

PCS : profession et catégorie sociale. 
Les étudiants peu privilégiés mettent tout en œuvre pour rentabiliser au mieux le temps passé à l'université, d'autant que leurs ressources ne leur permettent pas de se risquer dans d'autres voies, surtout si elles sont coûteuses. Mais c'est aussi une manifestation du nouveau visage des étudiants appartenant à la fraction la moins précarisée des catégories populaires. Ils aspirent à des études prolongées qui mènent notamment aux professions de la fonction publique, "véritables flières des mobilités populaires ascendantes par l'université » (Hugrée 2009, p. 53). Ainsi, comme les données qualitatives le mettent en évidence, les étudiants issus des milieux populaires ont plus de chance d'obtenir leur licence en trois ans que ceux de milieu favorisé. La régression logistique présentée dans le tableau 3 montre que cet effet est visible uniquement dans l'université la plus populaire (Fac Nord) et ne joue pas à Fac Ouest. Il est possible que la composition même de cette catégorie soit sensiblement différente dans les deux universités ; comme on l'a vu précédemment, la ségrégation socio-scolaire de l'AES est moins marquée à Fac Nord.

Le tableau 3 montre que l'effet le plus puissant sur la réussite aux examens reste le bagage scolaire, en particulier le type d'études secondaires aboutissant à un bac général. Ce résultat confirme certaines recherches (Convert, 2010). Nous avons également introduit le lieu de naissance comme illustration d'un ancrage territorial et de recrutement au voisinage de chacune des universités. Toutes choses égales par ailleurs, cette dimension n'a pas d'effet propre sur la réussite en L1 ou en L3. En revanche, l'université d'appartenance joue notablement, les étudiants de Fac Nord, à mêmes caractéristiques (sexe, age au bac, type de bac, PCS, lieu de naissance), échouent davantage que ceux de Fac Ouest ; ils ont 1,3 fois plus de probabilité de ne pas obtenir leur L1 en 2005-06 et 1,5 fois plus de probabilité de ne pas être titulaire de la licence en 2007-08(régression non présentée).

Une de nos pistes d'explication repose sur l'hypothèse que ce sont les conditions d'études, l'encadrement pédagogique, les exigences académiques dont la déclinaison est différente dans chaque établissement qui vont distinguer les processus de socialisation des étudiants. Nous avons donc cherché à comprendre, à travers les données qualitatives, si les conditions de socialisation des étudiants pouvaient expliquer cet effet établissement (Cousin, 1993). La construction de l'affiliation ${ }^{4}$ nécessite des supports de socialisation ${ }^{5}$ qui seront issus soit des parcours biographiques, soit de l'organisation universitaire. Les deux univer-

4. Alain Coulon (1997) précise trois étapes dans la construction du "métier d'étudiant » : la phase d'étrangeté, celle d'apprentissage et celle d'affiliation. Nos données montrent que les étudiants peuvent mettre parfois une ou deux années à parvenir à cette affiliation et non pas quelques mois comme le souligne l'auteur. S. Monfort fait le même constat dans sa thèse sur les étudiants de sciences et d'AES (Monfort, 2003) : "L'affliation est marquée par une aisance à l'égard des règles diverses et nombreuses qui organisent la vie sociale et intellectuelle du travail universitaire " p. 155. Nous faisons ici également référence à la notion d'affiliation utilisée par Robert Castel (1995) pour montrer la construction d'une appartenance sociale.

5. On peut se reporter à D. Martucelli (2002) qui pointe l'importance des supports pour faire face à l'expérience d'un nouveau monde social où l'individualisation devient une épreuve nécessitant un processus de socialisation secondaire. Les supports ne sont pertinents que s'ils permettent de réduire les incertitudes de ce processus de socialisation. 
sités n'offrent pas les mêmes supports Nous distinguons deux registres récurrents dans les entretiens des étudiants : des supports issus de la sociabilité entre pairs et des supports issus de l'organisation universitaire, à la fois administrative et pédagogique.

À Fac Nord, la sociabilité, notamment en première année, est souvent fondée sur une homologie culturelle, et plus précisément une commune expérience de la migration dans leur trajectoire. On comprend dès lors, comme le souligne E. Maurin, que l'homogénéité sociale peut fabriquer un " entre soi " aux effets protecteurs, notamment pour atténuer les effets des conditions socio-économiques et familiales souvent précaires et parfois chaotiques (naissances précoces, décès parentaux, violences familiales, logements insalubres...). Lorsque les étudiants quittent cette université, ils gardent le souvenir de cette commune expérience de la vulnérabilité et prennent alors conscience de l'homogénéité sociale et culturelle dans laquelle ils évoluaient. Pour autant, si cette sociabilité protège et amortit certains effets de violence symbolique liés aux situations pédagogiques à l'université, elle ne permet pas toujours l'affiliation à l'organisation universitaire. Compte tenu de leur distance sociale avec ce "nouveau monde ", ils doivent trouver des supports extérieurs à leur groupe d'origine. Des échanges avec des enseignants peuvent alors s'avérer déterminants de leur trajectoire.

"Dans les amphis, on se retrouvait ensemble avec les amis du Mali mais on ne comprenait pas ce quil fallait noter. Il y avait toujours une bonne ambiance mais un bazar dans les emplois du temps et on ne pouvait pas avoir les informations pour mieux comprendre. " (Etudiant, L2, redoublant, AES, bac techno)

"Moi je vais à la fac avec plaisir, on se retrouve à la cafet, on se raconte ce qu'on vit mais dans les cours, je mennuie, je vois pas où le prof veut en venir. Pourquoi on nous donne pas un plan de cours? Ma cousine, elle est ailleurs et on lui donne un document pour expliquer comment ça marche " (Etudiante, L1, AES, bac ES)

À Fac Ouest, la sociabilité entre les étudiants est souvent moins fusionnelle ou moins affective et les étudiants expriment des relations plus distantes : "Je ne vais pas à la fac pour voir les copains parce que mes amis sont ailleurs mais on se rend des services, on échange mais que sur des sujets de la fac. C'est comme si cétait des relations de travail, on ne fait pas la fête mais on travaille. » (Etudiant, M1 GRH, ex AES, bac techno)

Dans ce contexte, les étudiants d'origine populaire s'habituent à vivre au sein de deux mondes, souvent clivés, mais disposant de leur régulation spécifique. Leur monde d'origine, avec leurs amis et leur famille, et le monde de l'université. "Je ne parle jamais de ce que je vis à l'université chez moi et c'est pareil pour les amis à la fac, je leur parle pas de ma famille. Ça ne sert à rien, ça n'apporte rien, c'est un peu comme avoir deux vies différentes. A la fac, c'est un peu comme un travail où on ne parle pas de ses histoires. " (étudiante AES, Fac Ouest, L3) 
L'organisation des enseignements a également une incidence sur les conditions de socialisation. Le volume horaire des enseignements se répartit entre les travaux dirigés et les cours magistraux. Favoriser les premiers, permettant des travaux de groupes, offre des circonstances de rencontres entre des étudiants de différents mondes sociaux, d'autant plus riches que la répartition sociale est hétérogène. Comme le souligne cette étudiante, «Moi, je sais que je ne suis pas aussi bonne que les autres à l'écrit mais je sais aussi que toute seule je ne peux pas progresser, j'ai besoin de travailler avec d'autres, de savoir comment les autres font" (Etudiante, L3, bac ES). "On échangeait beaucoup entre nous. C'est peut-être le travail en groupe qui nous a permis de nous lier les uns avec les autres. On était un petit groupe. Cela a facilité le travail. " (Etudiante, L3, AES, bac ES)

\section{Conclusion}

Notre propos initial était de mettre à jour deux formes de ségrégations, territoriale et universitaire, de voir si elles se renforçaient l'une l'autre et, enfin, d'examiner de quelle façon elles exerçaient un effet sur les parcours étudiants. Ces interrogations participent à une réflexion plus large sur les conséquences de la mixité ou de la ségrégation sociale sur les parcours de formation. Le contexte territorial détermine en grande partie les caractéristiques des populations étudiantes accueillies par les universités, mais au fil du cursus, un processus d'homogénéisation scolaire s'opère par l'élimination des étudiants à faible capital scolaire. De ce fait, les différences inter-établissements s'amenuisent sur ce plan, mais elles persistent s'agissant de l'appartenance sociale des étudiants. Enfin, pour les étudiants des milieux populaires, la différenciation sociale permet à une petite élite de se maintenir dans une filière pourtant chargée de stigmates négatifs. C'est au contact de ces étudiants que les plus éloignés des normes culturelles, et les plus marqués par un système qui reproduit les inégalités sociales, trouveront des supports de socialisation pour maintenir leur engagement dans les études, à la condition néanmoins d'avoir fait leurs preuves au cours de leur parcours scolaire.

\section{Bibliographie}

Albouy V., Tavan C. (2007), « Accès à l'enseignement supérieur en France : une démocratisation réelle mais de faible ampleur ", Economie et Statistique, ${ }^{\circ} 410$, pp. 3-22.

BeAud S. (2002), 80\% au Bac, et après? Les enfants de la démocratisation, La Découverte.

Beaud S. (2008), "Enseignement supérieur : la "démocratisation scolaire" en panne ", Formation Emploi, n 101, pp. 149-164.

Bodin R., Millet M. (2011), «L'université, un espace de régulation. L'“abandon” dans les 1 ers cycles à l'aune de la socialisation universitaire ", Sociologie, nº 3, vol. 2, pp. 225-242. 
Castel R. (1995), Les métamorphoses de la question sociale, Fayard, Paris.

Convert B. (2010), "Espace de l'enseignement supérieur et stratégies étudiantes ", Actes de la Recherche en sciences sociales, $3, \mathrm{n}^{\circ} 183$.

Coulon A. (1997), Le métier d'étudiant. L'entrée dans la vie universitaire, PUF.

Cousin O. (1993), "L'effet établissement. Construction d'une problématique ", Revue française de sociologie, vol. 34, pp. 395-419.

Dubar C. (2010), La socialisation, construction des identités sociales et professionnelles, A. Colin, nouvelle édition.

Dumoulin C., Filhon A. (2008), "Construire l'université de masse. Genèse de la filière AES », Colloque Ce que l'Ecole fait aux individus, Nantes, 16-17 juin.

Duru-Bellat M., Kieffer A. (2008), « Du baccalauréat à l'enseignement supérieur en France : déplacement et recomposition des inégalités ", Population, 63 (1), pp. 123-158.

Euriat M. et Thélot C. (1995), " Le recrutement social de l'élite scolaire en France. Evolution des inégalités de 1950 à 1990 ", Revue française de sociologie, 36 (2), pp. $403-438$.

Felouzis G. (2001), «Les délocalisations universitaires et la démocratisation de l'enseignement supérieur ", Revue Française de Pédagogie, n 136, pp. 53-63.

Filhon A. (2010), « La première année en filière administration économique et sociale : motivation, abandons et attentes des étudiants ", Formation Emploi, n 111, p.19-33.

Grafmeyer Y. (1966), Sociologie urbaine, coll. « 128 », Nathan.

Hugrée C. (2009), "Les classes populaires et l'université : la licence... et après ? ", Revue française de pédagogie, $\mathrm{n}^{\circ} 167$, pp. 47-58.

Lemaire S. (2007), "Les inscriptions à l'université : quel bilan ? " MEN-DEPP, Note d'Information 07.10.

Lemaire S. (2010), "Que deviennent les bacheliers après leur bac ? Choix d'orientation et entrée dans l'enseignement supérieur des bacheliers 2008 ", MEN-DEPP, Note d'information, $\mathrm{n}^{\circ} 10.06$, juillet.

Lemaire S. (2012), "Les parcours dans l'enseignement supérieur : devenir après le baccalauréat des élèves entrés en sixième en 1995 ", MEN-DEPP, Note d'information, $\mathrm{n}^{\circ} 12.05$.

Martuccelli D. (2002), Grammaires de l'individu, Paris, Folio, coll. « essais ».

Maurin E. (2004), Le ghetto français, Enquête sur le séparatisme social, Ed. du Seuil, « La République des idées ». 
Merle P. (2000), «Le concept de démocratisation de l'institution scolaire : une typologie et sa mise à l'épreuve », Population, 55 (1), pp. 15-50.

Merle P. (2002), « Démocratisation ou accroissement des inégalités scolaires ? L'exemple de l'évolution de la durée des études en France (1988-1998) », Population, vol. 57 (4-5), pp. 633-660.

Ministère de L'EduCATION NATIONALE ET MiNisTÈre DE L'ENSEIGNEMENT SUPÉRIEUR ET DE LA ReCHERCHE (2007), Repères et Références statistiques sur les enseignements, la formation et la recherche.

MontFort V. (2003), Les étudiants de première année à l'université et le travail scolaire, l'exemple de deux filières : Sciences et AES, Doctorat de sociologie, EHESS.

Nicourd S., Dumoulin C., Filhon A., Samuel O., Vilter S. (2009), Trajectoire d'études et d'insertion sociale des étudiants d'AES. Suivi de cohorte d'étudiants d'AES dans deux universités, Rapport de recherche, Laboratoire Printemps, 100 p.

Prost A. (1986), L'enseignement s'est-il démocratisé ?, Paris, Puf.

SAUtory O. (2007), "La démocratisation de l'enseignement supérieur : évolution comparée des caractéristiques sociodémographiques des bacheliers et des étudiants ", Education \& formation, $\mathrm{n}^{\circ} 74$, pp. 49-64. 